\title{
Remote CAN Operations in MATLAB over the Internet
}

\author{
Sven Rönnbäck, Kalevi Hyyppä, Ake Wernersson
}

\begin{abstract}
This paper describes the implementation of a CAN server that acts as a CAN tool to a client. It can be used to monitor, observe and send messages to a distant CAN network over IEEE802.11b (Wave-LAN). The CAN server is controlled by one or several clients that can connect to it by TCP/IP. It is possible to send and receive CAN messages over Internet from a MATLAB environment since the client software is written in Java. The CAN server collects CAN messages and stores them into a ring buffer. The messages in the ring buffer are classified by their identifier and stored into a database. The CAN tool has been used in a demonstrative application example that consist of a remotely controlled wheelchair. In the example the wheelchair was programmed to run in a square. The positions obtained by odometric CAN messages are compared with the position from the navigation system onboard the wheelchair.

Index Terms- MATLAB, CAN-tools, Remote CAN operations
\end{abstract}

\section{INTRODUCTION}

In the early 1980's the Robert Bosch GmbH[1] company invented the Controller Area Network (CAN) to meet real time transfer requirements in the automobile industry. It can operate up to $1 \mathrm{Mbit} / \mathrm{s}$ and has good error detection. CAN is a distributed network with no central unit and is flexible in size. It is a good design for nodes that sends information in bursts. Messages sent by one node are broadcasted to all other nodes. It can be used in real time task since an identifier with lower number automatically gets higher priority on the bus. CAN is often used to connect micro-controllers over a simple network. CAN frames has an 11- or 29-bit identifier and a payload of maximum eight data bytes. If a collision is detected by a node during the send process the node looses its arbitration and tries to send the message after a delay. All nodes acknowledges frames by a flag and any node flags if a transmit error occurs. The MICA (Mobile Internet Connected Assistant) project has a modern wheel chair that is equipped with a CAN bus. The idea is to implement navigation algorithms in MATLAB using camera modules and other CAN nodes on the wheel chair as sensors[2]. Each camera module will report distance and angle to beacons, coded in a CAN message. We operate the CAN bus from the MATLAB environment by a CAN Java client that has a TCP/IP client/server solution that works over Wave-LAN.

MATLAB comes with a Java Virtual Machine (JVM) that can execute Java byte code in any MATLAB script.

There exists lots of different software and hardware solutions to poll and send information to the CAN bus from a PC (Personal Computer). The CANbus ${ }^{T M}$ toolset can

S. Rönnbäck, K. Hyyppä and $\AA$. Wernersson are with the Department of Computer Science and Electrical Engineering, Lulea University of Technology. S-971 87 Luleå, Sweden. (email $\{$ sr,kalevi,akewe\}@sm.luth.se) together with the appropriate hardware be used to create an interface between MATLAB and CAN bus[3]. This hardware has no Wave-LAN support so it is difficult to remotely analyze CAN messages from a moving vehicle. That product provides no solution to our project.

TCP/IP (Transmission Control Protocol/Internet Protocol) is communication peer-to-peer. TCP/IP does not have a deterministic delivery time. Theoretically, the delivery time of a message can be unbounded if there is a collision at each attempt to send the message, so WaveLAN under heavy traffic is a risky solution for a real- time network. If a collision is detected a Wave-LAN node backs off and tries to send the package after a short period of time[4], [5], [6], [7].

The IEEE802.11b (Wave-LAN) is somewhat unreliable when terminals or nodes lose and re-acquire line of sight very suddenly[7].

When a Wave-LAN node receives a packet to be transmitted, it first listens to ensure no other Wave-LAN node is transmitting. It transmits the packet if the channel is clear, else it backs off and randomly selects the amount of time the it must wait until it retries to transmit the packet. The back-off factor is selected in such a way that the probability for two nodes to get the same factor is low. Collision detection, as in Ethernet, can not be used for Wave-LAN since a node is deaf when it transmits data.

TCP on Wave-LAN guarantees the delivery of all data but not the delay or the rate of delivery[8].

Carnegie Mellon University (CMU) has a project with the name ROSES (Robust Self-Configuring Embedded Systems) that seeks methods for flexible, robust systems with built in graceful degradation that improves operational availability of an embedded system. Within this project they built a system that remotely reads CAN data from an automobile[9].

Our CAN server works almost like the one in the ROSES project. It gives us the possibility to remotely $\log$ and analyze CAN data on line.

\section{A. The Wheel Chair and its CAN bus}

The MICA wheel chair shown in Figure 1, is a research platform equipped with a CAN bus and an embedded PC. The CAN bus operates at $250 \mathrm{Kbits} / \mathrm{s}$ and is the common communication link for the different modules such as the joystick and the main micro-controller. The wheel chair has a manual control unit with setup facilities. The joystick makes it possible for an able user to control the wheel chair with high precision. The vehicle has incremental encoders mounted on the shaft of each front wheel. They are used as feedback to the micro-controller unit.

The micro-controller controls the translation and rotational velocities of the wheel chair. It listens to the CAN 


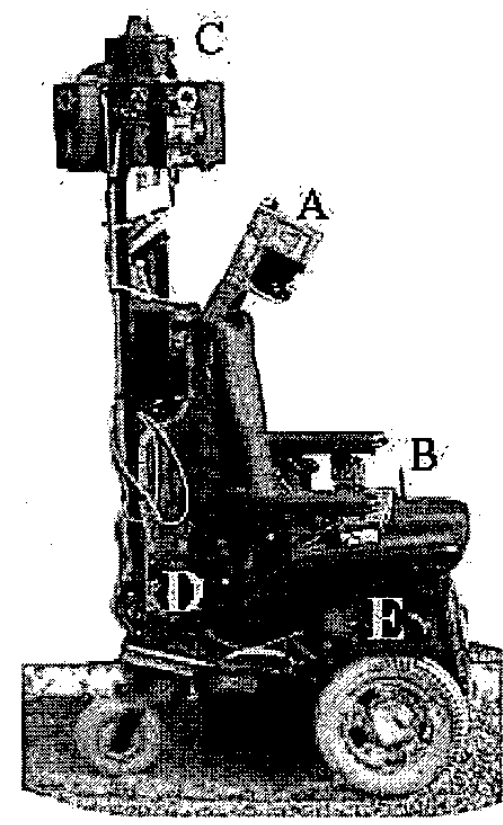

Fig. 1. The MICA wheel chair. Four flash cameras connected to the CAN bus are mounted below the LazerWay navigation system on the top (C). The IEEE802.11b access point is placed on the seat (B). The embedded PC computer (PC104), with the CAN interface, is mounted at position (D).

bus for driving commands and broadcasts encoder information.

The embedded PC (Personal Computer) is called the PC104 since it is built on the PC104 stack architecture. The mother board is a Pentium II class system that runs Red-Hat Linux with kernel 2.4.20 and the Real Time Application Interface (RTAI) patch.

The camera sensor modules send out infrared flashes and detect reflective tapes after image processing which is done in custom built hardware. This will produce estimates of angle and distance to reflectors which are streamed out on the CAN bus[10].

Each sensor is an independent module that can be added or removed to the system, Figure 2. We need electrical power and an interface to be able to use it. On the PC104 we start a program that communicates with the sensor device and acts as a server. About 50-600 CAN messages per second are collected by the CAN server. Most of them are time ticks, drive commands, reflector estimates from the flash cameras, messages generated by the LazerWay[11], [12] navigation system, messages with incremental encoder information and messages from the manual control unit.

\section{The CAN SERVER}

When the server program starts, it directly starts to collect messages from the CAN hardware and store them in a database, Figure 3. CAN messages are added as long as the PC104 still has unallocated memory or the maximum size of the database is not reached. If the maximum size is reached the oldest information is released and re-

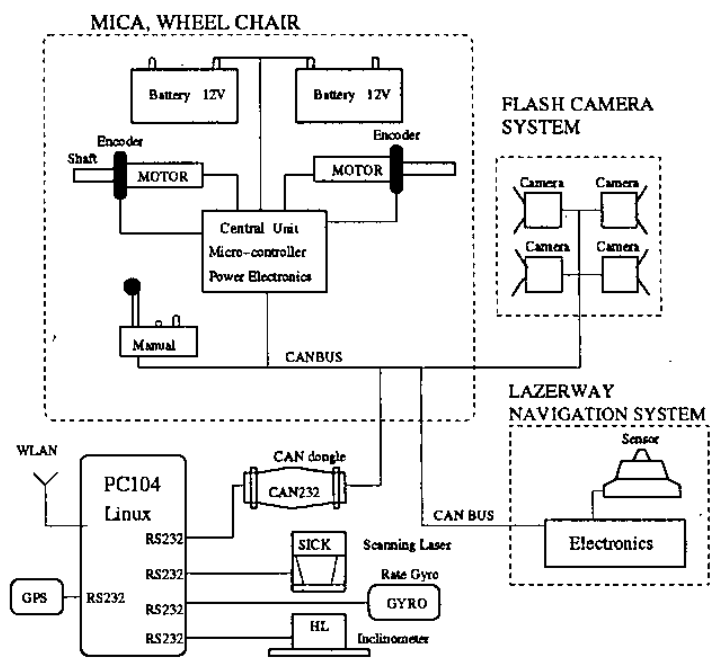

Fig. 2. Two powerful electrical motors drive the wheel chair. Using a joystick it is possible for the operator to control the wheel chair. Commands from the manual control unit (joystick) or the PC104 are sent over the CAN bus to the micro-controller that executes them and controls the motors on the wheel chair. On the shaft of each motor an incremental encoder is mounted. The encoders are read by the micro-controller and odometric information are streamed to the CAN bus. The cameras process the images and stream out the estimated position and distance to reflector.

placed by new. This is done with a ring buffer. When the maximum size of the ring buffer is reached it wraps around and starts from the beginning. We can easily with this system do our experiments, store all measurements in the database and after a test recall the data into the MATLAB environment. We need to know the start time and end time of the test or the indexes to recall the data needed. If we want to be sure that our measurements will be kept in the database we can stop the collection of new data.

\section{A. The CAN Server Threads}

The server runs several threads. In the beginning three threads are running. One thread that communicates with the CAN232 interface [13], the dongle time stamps the CAN messages as the are read from the CAN bus. The polling thread reads messages from the CAN232 dongle and puts them into a database, Figure 4. After each poll the status flag is read to check for errors.

In the database, Figure 4, the incoming messages are classified with respect to the identifier. For every new identifier a new list is created and added to the database. The list has a set of messages with the maximum length of 10000 messages by default. Encoder information is streamed onto the bus 40 times per second. So the list with encoder values will be full after about 250 seconds, however the maximum list length can be set to an arbitrary number during operation. CAN messages older than 15 minutes will automatically be deleted. If there exists four unique CAN identifiers there exists four different CAN message lists, as shown in Figure 4.

It is also possible to create lists that trigger on the iden- 


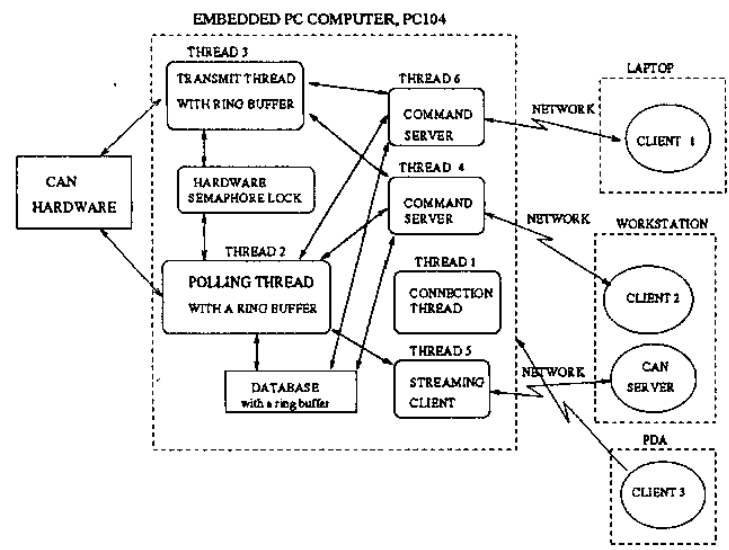

Fig. 3. The CAN server on the PC104. The CAN messages collected and stored in the database by thread 2 . Thread 1 is created first and waits for clients to connect. For each connected client a new thread is created, e.q. thread 4 and 6 . A streaming thread (thread 5 ) is created by thread 1 after a request from client 2 and is used to send incoming CAN messages continuously to the client. Client 3 is just about to connect to the server. A command thread executes commands sent by the connected client and sends the result back to the client. When a client disconnects the corresponding thread on the PC104 will terminate automatically.

tifier by Boolean algebra using code and mask registers[1].

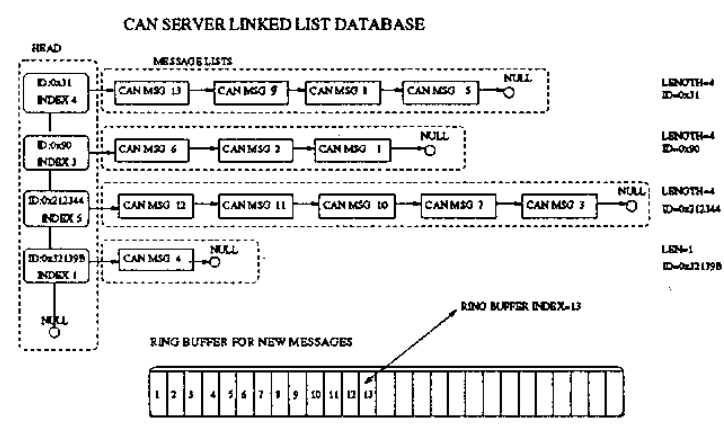

Fig. 4. View of the linked list structure used in the CAN server database. This example has a head list that holds four different CAN identifiers. The first identifier has four stored messages in the tail. On the bottom the ring buffer, that keeps a copy of the latest messages received, is shown.

The server software keeps track on encountered errors and the number of sent and received messages.

A connected client can put itself as a master, commands from other clients will then be ignored. This option is implemented for safety reason so that a human can overtake the automatic control with a remote control unit.

The server also has a message transfer database. CAN messages the clients want to send can be placed in a list. The client sets the start time when the message should be sent at the first time, its duration and repetition frequency. The message will stay in this database as long as the duration option imposes or until it is deleted by a command.

A fourth thread checks for connecting clients. When a connection is made a new thread is created to serve the requests from the client.

There is no upper limit on the number of clients that can connect, except for the limit set by the amount of memory.

\section{B. The CAN client}

Our CAN client is written in Java. We decided that MATLAB is a good environment to develop software and algorithms in. MATLAB is not a fast executing language since it interprets the program lines. However we speed up the development cycle (planning, coding, testing and analyzing) by using MATLAB. Sensor information is directly available over Wave- $\mathrm{LAN}$ as the server in the $\mathrm{PC104}$ buffers measurements. In MATLAB we use the Java client to connect to the CAN server since Java can run in that environment. Java code is a portable language and it is very easy to reuse code in other applications and environments. The client instance has a lot of methods that can be used to manipulate, poll and send messages to the CAN server. The connect method is used to make a TCP/IP socket connection to the CAN server. As the connection is made a new thread is created to serve requests from the client.

\section{B.1 A MATLAB script that polls CAN messages from the CAN server}

An illustrative MATLAB script that dumps CAN messages to the screen.

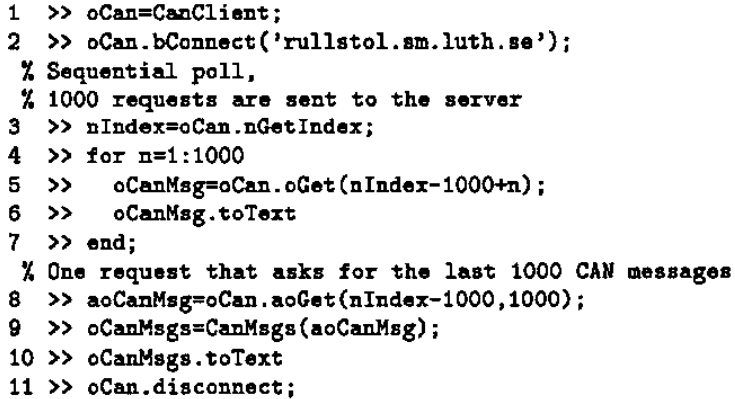

The MATLAB script opens a TCP/IP connection to the server, line 2 . On line 3 it sends a request to the server for the current cyclic buffer index. Line 4-7 are used to poll 1000 CAN messages and print them as text on the screen. Line 8 asks, in a single request, the server for an array of $1000 \mathrm{CAN}$ messages. Line 9 encapsulate the CAN array in an object. Line 10 calls the toText method which dumps all the 1000 CAN messages as text to the screen.

In the two given examples above we polled CAN messages from the ring buffer by using the ring buffer index. It is also possible to request CAN messages by their identifier or by the time stamp.

In the developing process of new algorithms that uses the CAN interface, it can be very useful to get statistics about the CAN server. The statistics are directly available through a method in the CAN client and on the WWW.

With the CAN client it is possible to drive the wheel chair and collect odometric and camera sensor information that we need for data fusion. The LazerWay navigation system is also accessible trough the CAN interface, by this 
we can compare our navigation algorithms with a known working system.

\section{B.2 MATLAB script that sends a CAN message to the CAN server in two different ways}

To send a CAN message in MATLAB we create a message instance and send it using the CAN client. An illustrative example how this can be done follows below:

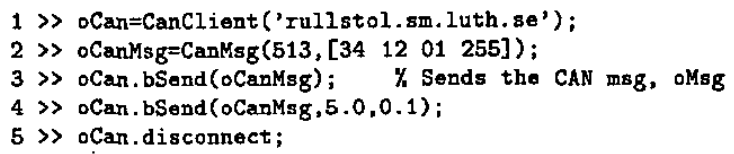

Line 1 connects the CAN client to the CAN server on the wheel chair. A CAN message with the identifier 0x201 and data field $([0 \times 220 \times 0 C 0 \times 010 \times F F])$ is created in line 2. On line 3 the CAN message is sent over Wave-LAN to the wheel chair using the bSend function that blocks and returns a Boolean value of the operation. On line 4 we send the message using the send database in the server by adding two extra arguments. They say we want to send the message for 5.0 seconds every 0.1 second. Line 5 disconnects the client from the CAN server.

\section{B.3 Some CAN Server Commands}

We have implemented some functions and command that make it possible to operate the CAN server over TCP/IP. All received messages on the server get a unique increasing index and are time stamped using the system clock. When 100 messages have been received the index is 100 in the ring buffer. Some of the implemented CAN functions are listed below:

Get system time: Returns the system time on the server. It is taken form the clock and used to time stamp messages.

Get database status: Returns information about number of collected and transmitted messages. The number of different types of messages with respect to identifier and the number of messages for each identifier.

Search for ring buffer index using time: It is possible to search for the ring buffer index for message stored in the ring buffer.

Send immediately: Send a message directly to the CAN bus without using the transfer database.

Get status: Returns the client status and statistics about the CAN server, the number of connected clients, the number of errors encountered, the number of messages received and sent and the number of messages in the receive database and transmit database. It will also send a list of different messages sorted with respect to CAN identifier.

Get message by index: The client asks the server for a message with a specific index. If this index is bigger then the ring buffer index the server will block until that index is reached. If the requested index is zero the most recent message will be sent back to the client.

Get messages by identifier and time: Returns an array of message objects with an specific identifier. The array has a selected start and end time.
Get message by identifier and list index: The command returns the message with the desired index from the list holding the messages with a specific identifier.

Send message through transmit database: A command used to send messages with the transmit database. It is possible to set the duration of the message, with what frequency it should be broadcasted and the time when the first transmission starts.

Remove message from transmit database: Removes a message from the transmit database.

Get index: Return the index for the most recent message in the database.

Set code/mask filter: Makes it is possible to set both the code and the mask registers in the controller chip. It is used to mask out messages on the CAN bus using the hardware.

Set software code/mask filter in the database: All received messages that are not masked away will be inserted in a message list. This command is very useful to collect all messages that belong to the drive unit such as maneuver commands and messages with wheel encoder information. The wheel chair has two different encoder messages. One with absolute encoder values and one with differential encoder values, both of them can be masked into the same list.

Send message and wait for identifier: Sends a message to the CAN bus and waits for a special identifier. It is possible to mask the answer with the implemented software filter that works like the acceptance filter used in the CAN controller, SJA1000 by Philips. The acceptance filter compares the received identifier with the acceptance filter values, and decides if the message is valid or not. The acceptance filter can be described using Boolean algebra. If the following statement is true we have filtered away a correct identifier and a 11 bit CAN message will be accepted: NOT ((ID XOR CODE) OR MASK) XOR $0 \times 3 \mathrm{FF}) \in\{$ false, true $\}$

In the list above some commands were mentioned which poll the server for messages or message arrays using the index. It is also possible to use other commands to poll the server for CAN messages from a selected start time and end time. These commands are very useful when experiments are done since we can poll the server for messages using a negative time value, the server will then give us the CAN messages that were polled under the experiment. A time value less then 100000 are treated as an offset from the current time, this means that 1000 will result in a wait for 1000 seconds.

\section{B.4 CAN Server HTTP Interface}

The CAN server also gives the user an opportunity to check the CAN statistics using a WWW browser using HTML code. It is possible to check both the incoming and the outgoing message queues.

The incoming CAN identifiers are listed in ascending order with the possibility to check for the last read messages in an identifier list. It is easy, through the WWW page, to check for errors and different message identifiers in the message database. This is very useful in a debug- 
ging process when we quickly can check the incoming and outgoing databases.

\section{CAN Server Database Status as Text}

In MATLAB it is neat to get a view of the different messages in the CAN database. It is therefore possible to call a function that polls the CAN server for database information that returns a Java instance. The instance contains the topic of each identifier list: The identifier, current index and length of each list. In the list we also see the most recent received message for that list, presented as the topic.

\section{Can Bridge Over TCP/IP Using the CAN Servers}

It can be useful to connect physically isolated CAN networks over some network A program was coded in Java that mutually checks and polls each CAN server for new messages and sends them to the other network. It cannot guarantee real time but it is a solution that gives a system where all messages sent by a CAN bus node in one network appear in the other network. The CAN server works as a link between the CAN bus and the network. The CAN@net from Nohau [14] is a CAN Ethernet gateway. This product has a program interface that works in the Windows operating system.

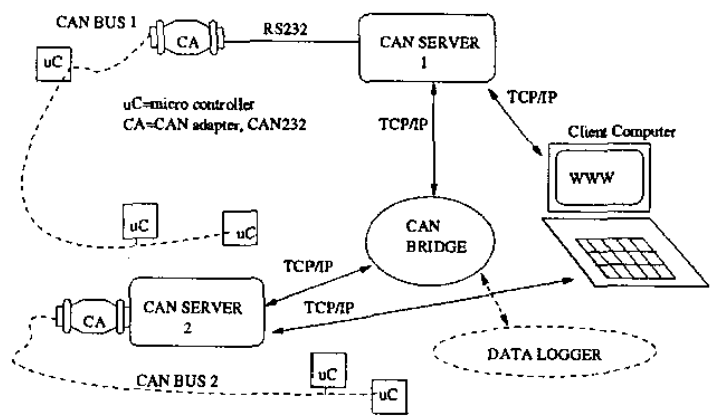

Fig. 5. The "CAN BRIDGE" Java program acts as a bridge between two physically isolated CAN networks. Server 1 has a remote connection to the CAN adapter (CA) using a serial link connection over the Internet and TCP/IP protocol. This makes it possible to use the CAN232 on a very tiny computer with lack of memory while the information is streamed back to the server. With the CAN bridge, micro-controllers (uC) on different buses can broadcast messages to each other.

\section{E. Time Stamps and Time Synchronization}

Received CAN messages are time stamped with the system clock of the PC104 onboard the wheel chair. Since $\mathrm{PC} 104$ and the other computers in use are time synchronized, using the Network Time Protocol (NTP), the system time difference between two computers is small. Messages send to the the wheel chair are time stamped with the system clock. The time difference between the system time and time stamp in a received CAN message can be used to indicate network lags or time jitter. It is important to have this in mind at since TCP/IP has no deterministic delivery time on either IEEE802.11b or Ethernet.

\section{RESULTS}

The example shown in Figure 6 uses the Java CAN client in MATLAB to plot the accumulated position of the vehicle using CAN messages with encoder information. It is a illustrative example showing how easily CAN information can be accessed from MATLAB.

In the example a set of telecommands were sent to the wheel chair navigation system that makes the vehicle move in a square. The sequence is:

Move forward $1.45 \mathrm{~m}$, stop and turn $90^{\circ}$ to the left.

Move forward $0.8 \mathrm{~m}$, stop and turn $90^{\circ}$ to the left.

Move forward $1.45 \mathrm{~m}$, stop nd turn $90^{\circ}$ to the left.

Move forward $0.8 \mathrm{~m}$, stop and turn $90^{\circ}$ to the left.

And it is back at the start.

The wheel chair uses the dead reckoning system to navigate based on odometric and rate gyro information. It was running for about one minute. The encoder information was broadcasted from the central micro-controller in the wheel chair with a frequency of $10 \mathrm{~Hz}$, which gives about 600 CAN messages. The CAN messages that has encoder information was polled from the CAN server and run through a loop to calculate the wheel chair position. The incremental encoder information were converted into differential odometric values.

A model for a differentially driven vehicle was used in the example. The wheel chair velocity expressed in vehicle frame $\{W\}$ at discrete time $t(k)$ is:

$$
v_{w}(k)=\left[\begin{array}{c}
v_{w x}(k) \\
v_{w y}(k)
\end{array}\right]=\frac{1}{2}\left[\begin{array}{c}
\omega_{l w}(k) r_{l w}+\omega_{r w}(k) r_{r w} \\
0
\end{array}\right]
$$

$v_{w}(k)$ : The wheel chair velocity expressed in wheel chair frame $\{W\}$.

$r_{l w}:$ The left wheel radius.

$r_{r w}$ : The right wheel radius.

$\omega_{r w}(k)$ : The measured angular velocity of the right wheel calculated from CAN messages with encoder information. $\omega_{l w}(k)$ : The measured angular velocity of the left wheel calculated from CAN messages with encoder information. $t(k)$ : Is the time obtained from the CAN message.

Angular velocity or turn rate $\left(\omega_{w}(k)\right)$, expressed in navigation frame $\{G\}$ of the wheel chair, calculated by the use of encoder information becomes:

$$
\omega_{w}(k)=\frac{\omega_{r w}(k) r_{r w}-\omega_{l w}(k) r_{l w}}{L_{w}}
$$

Where $L_{w}$ is the distance between the two front driving wheels. The estimated position and heading based on CAN messages was compared with the position obtained by the dead-reckoning system onboard the vehicle, that uses a rate gyro to stabilize the heading. Figure 6 shows a difference in position between position obtained by the $\mathrm{CAN}$ messages and the position from the dead-reckoning system onboard the wheel chair. We can see that the estimated position of the wheel chair based on CAN messages drifts to the right. The reason may be lower wheel pressure on the right wheel.

The drift in heading is estimated to be about -0.2 degree/s, Figure 7 . The wheel chair moves along the side 
and at each corner it stops and turns $90^{\circ}$.

The vehicle starts to make a turn at $t=10$ second. In the Figure 7 we can see that there is a lag between the CAN message heading calculation and the gyro heading calculation indicated by the periodic increase and decrease of heading. It is hard to tell where this lag occurs, but we suspect that it is the time difference between the rate gyro measurements and the odometric CAN messages.

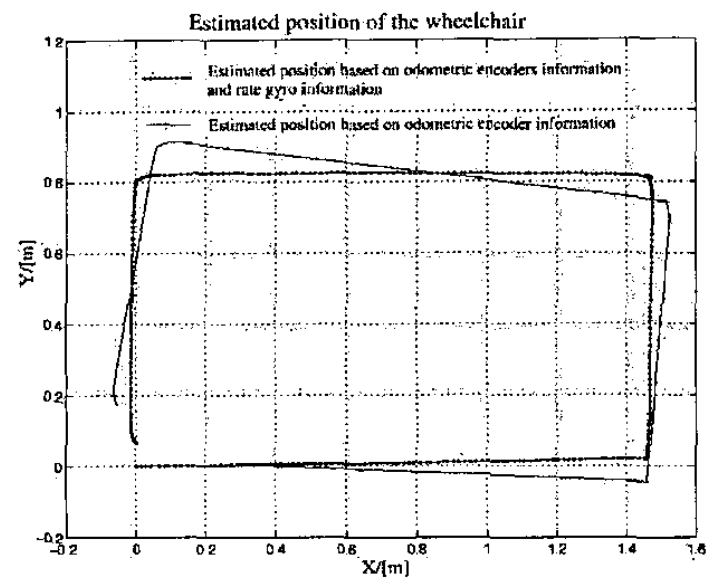

Fig. 6. The estimated wheel chair path based on odometric information from CAN messages are compared with the path read from the dead-reckoning system onboard the wheel chair which has a fiber optic rate gyro to stabilize the heading.

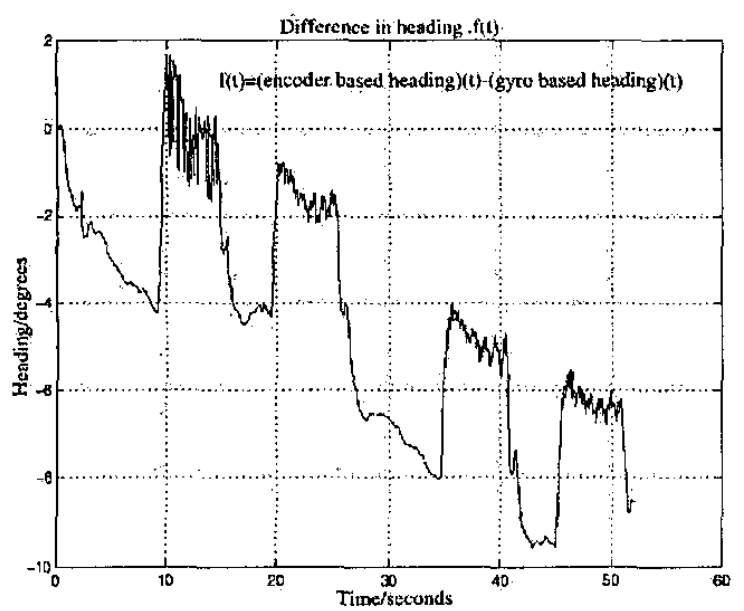

Fig. 7. The difference in heading between the heading based on odometric information from CAN messages and the heading from the onboard dead-reckoning system which uses a fiber optic rate gyro to stabilize the heading.

\section{Conclusions and Future Work}

We have a CAN server that gives us remote CAN operation. The MICA wheel chair has a sensor and actuator environment that is now accessible from MATLAB.

The MICA wheel chair has been remotely driven by telecommands from MATLAB. Sensor information is available over TCP/IP in a client/server solution.
The received CAN messages are put into a database that has a cyclic array where the most recent messages are kept. It sorts the messages by the identifier or by user selected masks.

Several clients can connect to the CAN server and send and receive messages concurrently. The client trigs on special events such as a unique index, time or CAN identifier.

We will also introduce a password restriction in the server so that a connected user must login before he can use the CAN server.

We will implement receiving threads in the clients, so that data and measurements in the server databases or ring buffer will be transmitted to the client side automatically. A mirror of the data selected could then be accessed in the client database. The polling is a time consuming process due to network delay. If we had a client mirror database the CAN messages could be found almost directly and network delay can then almost be neglected. However there is a maximum bandwidth on the network and the wireless network bandwidth is currently bound to $11 \mathrm{Mbit} / \mathrm{s}$. The continuous stream of data to the client would use a lot of this bandwidth but it would still be a better solution than to sequentially poll data.

\section{ACKNOWLEDGMENTS}

Thanks to Lars Wictorsson at the company Lawicel for the CAN232. The MICA project is partly funded by Interreg IIIA Nord.

\section{REFERENCES}

[1] Robert Bosch, CAN Specification 2.0, Robert Bosch GmbH, http://www.can.bosch.org,2003, Sep 1991.

[2] Sven Rönnbäck, David Rosendahl, and Kalevi Hyyppä, "A matlab/java interface to the mica wheelchair," 1st IFAC Symposium on Telematics Applications in Automation and Robotics, pp. -, July 2004.

[3] MathPros, "Canbus toolset for use with matlab and simulink on pcs," http://www.mathpros.com, Dec 2003.

[4] Håkan Lindberg, Trädlōsa Nätverk, Studentlitteratur, ISBN 91-44-02696-X, 2002

[5] Bernhard H. Walke, Mobile radio networks : networking, protocols and traffic performance, Wiley, 2002.

[6] Hans Scholten, Pierre Jansen, Ferdy Hanssen, Wietse Mank, and Arjan Zwikker, "Benchmarking for $r$ tn, a real-time variation on ieee802.11," http://www.ub.utwente.nl/webdocs/ctit/1/000000d5.pdf, Feb 2004.

[7] P. Bergamo, M.Cesana, D. Maniezzo, G. Pau, K. Yao, $D$. Whiteman, and M. Gerla, "Teee 802.11 wireless network under aggressive mobility scenario," International Telemetry Conference ITC/USA, Oct 2003.

[8] James F. Kurose and Keith W. Ross, Computer Networking, Addison-Wesley, 2 edition, 2003.

[9] Ernie Pusateri, Chris Martin, Pratish Halady, and Aditi Bajoria, "Data collection for can-based distributed systems," http://www.cs.cmu.edu/afs/cs.cmu.edu/project/iceseris2/ roses, Dec 2003.

[10] Evensson M., Kozmin K., Marklund A., and Àhsberg K., "Ett kamerabaserat navigationssystem," M.S. thesis, Luleå University of Technology, 2002:091.

[11] Danaher-Motion Särö AB, "Danaher-motion," http://www.ndc.se, Sep 2003.

[12] LazerWay, "Lazerway," http://www.lazerway.com, 2004 Jan.

13) Lars Wictorsson, CAN232 Manual 2.0A, Lawicel, http://www.candip.com, Nov 2003.

[14] Nohau, "Can@net gateway module," http://www.nohau.se, Nov 2003. 